\title{
Asthma mortality in Danish children and young adults, 1973- 1994: epidemiology and validity of death certificates
}

\author{
I.M. Jørgensen*, S. Bülow*, V.B. Jensen*, T.L. Dahm*, P. Prahl*, K. Juel**
}

\begin{abstract}
Asthma mortality in Danish children and young adults, 1973-1994: epidemiology and validity of death certificates. I.M. Jørgensen, S. Bülow, V.B. Jensen, T.L. Dahm, P. Prahl, K. Juel. C ERS Journals Ltd 2000.

ABSTRACT: Several reports indicate that asthma mortality has increased during the last few decades. International comparisons reveal some striking differences in the pattern of asthma mortality. The authors investigated the asthma mortality rate in the Danish child and youth population 1973-1994 and studied the validity of death certificates.
\end{abstract}

The authors reviewed all death certificates coded as asthma death in the International Classification of Diseases (ICD 8-ICD 10 (1994)) and adjacent respiratory code numbers for the age group 1-19 yrs. Hospital records and autopsy reports were assessed to validate the cause of death. Age-standardized and age-specific mortality rates were calculated.

From 1973 to 1987 there was a significant upward trend in the mortality. On subdivision, this trend was limited to the age group 15-19 yrs. Generally the mortality rate decreased from 1988 to 1994 . Four per cent coded as asthma were false positive. Twelve per cent were false negative asthma deaths, wrongly coded as due to other causes. Only $62 \%$ of all true positive death caused by asthma were appropriately coded. The number of false negative certifications increased with increasing autopsy frequency.

Asthma mortality rates in Denmark increased in adolescents during 1973-1987 and decreased from 1988 to 1994. A possible explanation may be an increased awareness of asthma symptoms combined with a steadily improved treatment of asthma. Even in children and young adults under the age of 20 yrs, validity problems still make comparisons between countries difficult; even interpretation of national trends requires caution.

Eur Respir J 2000; 15: 844-848.

Several reports indicate that asthma mortality has increased during the last few decades [1-4]. Fatal cases among children are rare and epidemiological studies of asthma death in children and young adults are rare [5-7]. International comparisons reveal some striking differences in the pattern of asthma mortality $[1,2]$.

Comparison between countries can be difficult because the interpretation of mortality data is confused by, firstly, revision of the International Classification of Diseases (ICD-8) in 1979 in some countries. The new code, ICD-9, artificially increased the number of registered asthma deaths [8]. Until 1994 ICD-8 was used in Denmark for $>25$ yrs. In 1994 ICD-10 was introduced. Secondly, uncertain reliability of mortality data is also attached to national differences in interpretation of the ICD-coding and in definition of asthma death as underlying cause of death. Diagnostic transfer is a third factor which has to be considered in trend analysis. The clinical criteria for diagnosing asthma may have changed with time. It is generally accepted that for patients aged $<35$ yrs the accuracy of the asthma diagnosis on death certificates is clear $[8$, 9]. However, even in this age group and especially among children, the extent of false negative certification of
*Dept of Paediatrics, Gentofte University Hospital, Copenhagen, Denmark. **Danish Institute of Clinical Epidemiology, Copenhagen, Denmark.

Correspondence: I.M Jørgensen

Dept of Paediatrics

Section 5003, Rigshospitalet

National University Hospital

Blegdamsvej 9

2100 Copenhagen

Denmark

Fax: 4539777639

Keywords: Asthma

children

death certificates

diagnosis

mortality

trend analysis

validity

Received: August 41999

Accepted after revision January 122000 asthma as underlying cause of death (i.e., asthma deaths wrongly certified as due to other causes) is less well studied [10-12].

The purpose of this study was to investigate and review the asthma mortality rate in the Danish child and youth population in the period 1973-1994 to identify trends in the rate. The authors also studied the validity of death certificates, with the purpose of estimating the proportion, and possible trend, of false negative certification and coding of asthma death.

\section{Materials and methods}

All death certificates in the age group 1-19 yrs where asthma was coded as underlying or contributing cause of deaths that occurred in residents in Denmark between January 1, 1973 and December 31, 1994 were obtained from the Department of Health Statistics of the Danish National Board of Health. Deaths from asthma referred to deaths coded as 493 in ICD-8, and the codes J 45-46 for the year 1994, when ICD-10 was applied in Denmark from January 1. 
Copies of all death certificates under adjacent respiratory code numbers (ICD-8: 490-492, 466 and ICD-10: J40-44, J47, J20-21) were also obtained and checked to estimate possible asthma death wrongly coded. A total of 431 death certificates were obtained. In Denmark the death certificate is issued by a physician or a coroner. If the manner of death is "sudden and unexpected", as with the majority of asthma deaths in children and adolescents, the police have to be informed. In such cases the information on the death certificate is expanded with a summary of the patient's medical history and the circumstances of the death.

The Danish death certificate closely follows the international form. Causes of death are coded according to the rules and classification of the World Health Organization. The underlying cause of death is defined as: the disease which initiated the train of events leading directly to death. This cause should be entered on the lowest used line of Part I of the death certificate. This condition is selected for statistical coding. In addition to these general rules relating to the death certificates, there are rules that apply when certain specified diseases are reported on the same death certificates. Under the 8th revision rules death from asthma, but with mention of bronchitis, bronchiolitis or emphyysema were to be attributed to the other conditions.

National data identified 70 deaths where asthma was coded as underlying cause of death. Two respiratory paediatric physicians reviewed, independently and unaware of the coding on the death certificates, all 431 death certificates.

If the death certificates mentioned asthma or raised suspicion of asthma (bronchial hyperreactivity or wheezing symptoms, treatment with medication usually used for asthma or atopy) as cause of death or the information on the death certificate was not conclusive, hospital records, information from the general practitioners and autopsy reports were obtained and assessed to validate the cause of death. In Denmark all inhabitants have a personal identification number which is certified on the death certificate. This enables hospital admissions, general practitioner and autopsy reports to be identified by different national database systems. Hospital records were available in $87 \%$ of the cases. For the remaining 13\% the medical notes were elucidated by the postmortem examinations and the coroner's reports, which were available on all these cases. All of the information was then reviewed and assessed with particular reference to age, sex, certified underlying and contributing cause(s) of death, time and place of death, autopsy and the following aspects: features and duration of asthma, severity of asthma, long-term and ongoing medical treatment, and circumstances of the final illness. A total of 152 cases were reviewed in this manner. In 279 death certificates, the certificate and coding were accepted as valid without further investigation.

A panel of five paediatricians with experience in respiratory diseases determined whether the patient died as a result of asthma. If there had been an autopsy, a pathologist with special interest in respiratory diseases was consulted. These respiratory paediatricians agreed to use their routine diagnostic skills. At least three of the paediatricians and the pathologist had to give the same diagnosis before it was accepted as the true cause of death.

Cases (two patients) where diagnostic agreement was impossible were excluded. Where information for deter- mining the cause of death was insufficient, these cases (two patients) were excluded too. None of these four patients out of 152 cases were certified as having died as a result of asthma.

Six deaths were provoked by allergen immunotherapy. The authors decided to exclude these cases. The coded underlying cause of death on these death certificates was asthma (493.0) in four cases and bronchitis in two cases. All of the fatalities were caused by conventional vaccination with aqueous mould extracts. At that time allergen extracts were poorly standardized as for the total allergenic activity. Especially in mould extracts large batch to batch variations have been shown. These dangers of this method have lead to the fact that immunotherapy with mould extracts is not recommended and if necessary it should only be performed with depot preparations and not with aqueous ones.

The study was designed as a historical follow-up study in a dynamic population [13]. The study population was children and young adults aged 1-19 yrs, resident in Denmark during 1973-1994. Denominator data were obtained by using population estimates based on agespecific populations on January 1 for each of the years 1973 to 1994 supplied by the Danish National Bureau of Statistics. In the case of trend analyses, the populations on January 1 1973-1994 were employed for risk time determination. The risk times were employed as denominators in the calculation of the mortality rates and as observation times attached to the stratified analyses. First, the average mortality rate per million population was calculated. Age-standardized mortality rates per million population were calculated for the age group 1-19 yrs and age-specific mortality rates for the various age groups 14, 5-14, 15-19 yrs. The statistical analyses applied were: Mantel-Haenszel's analysis for cohort data, the Fisher exact test, and Chi-squared in a discrete Poisson distribution [14]. A 5\% significance level was employed.

\section{Results}

A total of 102 cases were identified (by the panel of physicians) as having died as a result of bronchial asthma or asthmatic bronchitis (seven cases). Asthmatic bronchitis is here defined as recurrent wheeze with interval symptoms, and deterioration of asthma symptoms in connection with death, in children $<3$ yrs of age.

The average mortality rate was 3.9 per million personyears. From 1973 to 1987 there was a significant upward trend in the mortality. The rate of mortality decreased significantly from 5.8 per million person-years in 19831987 to 1.3 per million person-years in 1993-1994 ( $\mathrm{p}<$ 0.05 ). The trend in the overall asthma mortality rates for 1973 to 1994 is shown in figure 1 .

The significant changes were limited to the age group 15-19 yrs. There was no evidence for an increase in asthma mortality in 5-14-yr-old children over the study period. In the 1-4 yrs age group, there was a slight, but nonsignificant, upward trend in the mortality during 19731992. The age-specific asthma mortality rates are presented in figure 1 .

The 102 cases where asthma was the cause of death were coded under the following underlying causes of death. In 63 cases, asthma was correctly diagnosed and coded. Bronchitis or emphysema were coded as underlying 


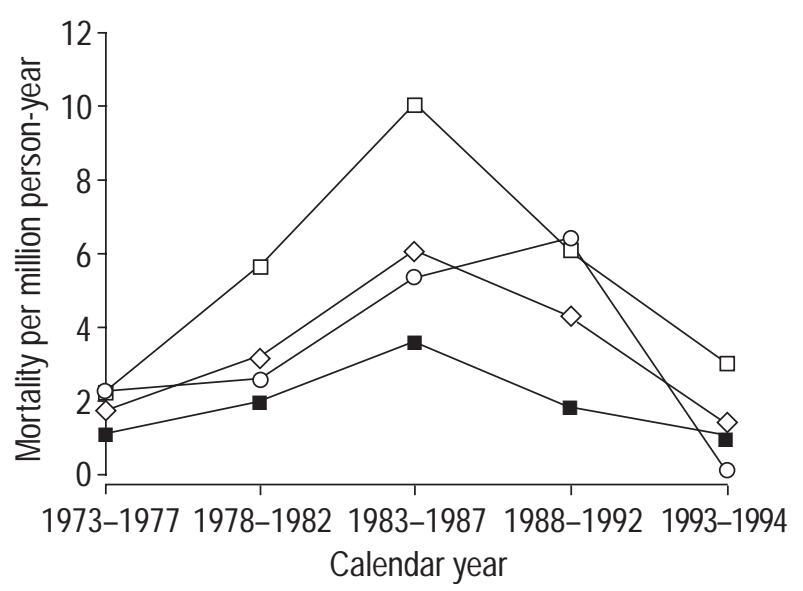

Fig. 1. - The overall mortality rates and age-specific mortality rates of death from asthma by calendar-year. Persons aged 1-19 yrs, Denmark, 1973-1994. $\bigcirc: 1-4$ yrs of age; $\square: 5-14$ yrs; $\square: 15-19$ yrs; $\diamond: 1-19$ yrs (overall). International Classification of Diseases (ICD)-8 and ICD-10 (1994).

cause of death in 31 cases. "Other causes" were cited as underlying cause of death in eight cases. These "other causes" were unspecified viral infection, cerebral infarct, pneumonia, medical intoxication and unknown causes. The coded contributing causes of death in these cases were as follows: asthma (five cases) and bronchitis (three cases).

The comparison of all the 152 death certificates and the hospital records/autopsy reports showed that $4 \%$ coded as asthma were false positive, i.e., deaths wrongly certified as due to asthma. Three per cent were false negative asthma deaths, i.e., asthma deaths wrongly certified as due to other causes. The proportion of false negative asthma deaths coded as bronchitis or emphysema was $9 \%$, and due to: deaths of persons certified as dying of asthma but with mention of bronchitis/emphysema were coded as being due to these causes. Only $62 \%$ of all deaths caused by asthma (as judged by the panel of physicians) were appropriately coded. The number of false negative asthma deaths varied during the study period and increased with increasing autopsy frequency. The trend in asthma mortality rates, by coded underlying cause of death, for 19731994 is shown in figure 2 . The percentage of correctly

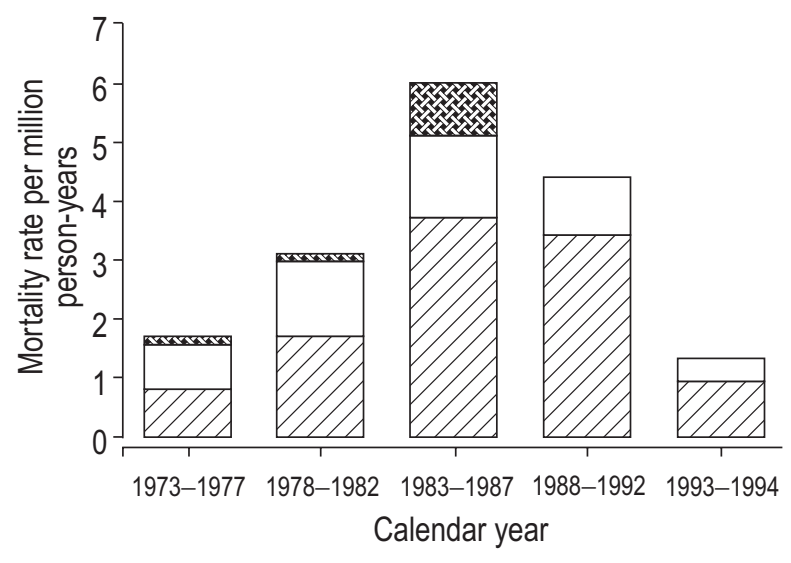

Fig. 2. - Mortality rates of death from asthma by coded underlying cause of death. Persons aged 1-19 yrs, Denmark, 1973-1994. $\mathbb{Z}$ : asthma 493; $\square: 490-492$; $:$ other causes. International Classification of Diseases (ICD)-8 and ICD-10 (1994). certified asthma deaths increased with age. Thirty-eight per cent were correctly certified as code 493 in the $1-4$ yrs of age group, $55 \%$ in the 5-14 yrs of age group and $75 \%$ in the $15-19$ yrs of age group. The proportion of false negatives was dependent on the age distribution. In the 1-4 yrs of age group, which represented $24 \%$ of the study material, $52 \%$ of these cases were certified under adjacent respiratory codes as 490-492. The 15-19 yrs of age group constituted $48 \%$ of the cases. Eighteen per cent were certified as 491-492.

As expected, the mortality rates calculated on ICD-8 code 493 (asthma) are lower than estimated in this study. The decrease in the asthma mortality rate starting in 1988 would not have been shown if no correction for false negative coding had been made.

The autopsy frequency decreased from $62 \%$ in 1978 1982 to $22 \%$ in $1988-1992$ and increased to $66 \%$ in $1993-$ 1994. Only three children died of asthma during this last 2yr period. The autopsy frequencies among the age groups were almost identical. The coded underlying causes of death by authority are shown in table 1 .

\section{Discussion}

It is a widespread belief that deaths as a result of asthma in children and young adults have increased since the early 1970 s $[1,3,5,7]$. This concern is based mostly on large age-aggregated national mortality database calculated studies $[1,2]$. A few studies have questioned this assumption $[15,16]$. The present study has shown a significant increase in asthma mortality in Denmark during 1973-1987. On subdivision, this trend was mainly limited to the 15-19 yrs of age group. The mortality rates in children under the age of 15 yrs were relatively stable. In Europe, this phenomenon is also described in the UK and Sweden $[5,15]$. The mortality rate for the 15-19 yrs of age group in Denmark during 1988-1992 was 6.0 per million person-years and approached the same level as in Sweden and the UK $[5,15]$. In England and Wales the asthma mortality rate in the 15-44 yrs of age group peaked before 1989 and then showed a downward trend during 1983-1995 [16]. The results were based on national database registered asthma deaths, ICD-9 code 493 [16].

An important finding of the present study is the decrease in the rate of death from asthma since 1988, especially among 15-19-yr-olds. During the 2-yr period 1993-1994, only three patients died as a result of asthma. In a country such as Denmark, where, generally, few observations of relatively uncommon afflictions can be made, a considerable amount of uncertainty is attached to the annual rates of

Table 1. - The coded underlying causes of death by authority

\begin{tabular}{lccc}
\hline $\begin{array}{l}\text { Coded underlying } \\
\text { cause of death }\end{array}$ & No autopsy & Medico-legal & Hospital \\
\hline Asthma 493 & 80 & 27 & 67 \\
490-492 (J448) & 14 & 55 & 33 \\
Other causes & 6 & 18 & - \\
\hline
\end{tabular}

All data are presented as percentages. Persons aged 1-19 yrs, Denmark, 1973-1974. International Classification of Diseases (ICD)-8 and ICD-10 (1994). 
death from asthma for 1993-1994. The last year for which mortality data in Denmark are complete is 1994. In 1995, no deaths due to asthma in persons under the age of $20 \mathrm{yrs}$ were registered (Danish Institute for Clinical Epidemiology). As mentioned, one limitation in using asthma mortality as an outcome is its relative infrequency. The advantage is a well-defined and important outcome, which is almost universally available [11].

In order to examine the validity of the death certificate diagnoses, the current authors collected all death certificates where asthma (ICD-8, 493) was coded as an underlying or a contributory cause of death. In an attempt to estimate the proportion of false negative asthma deaths, the death certificates where the underlying or contributing cause of death was statistically coded as bronchitis, emphysema or bronchiolitis were also considered. It was thought that this group probably included most of the unsuspected deaths that resulted from asthma. Hospital records, information from general practitioners and autopsy reports were assessed to validate the cause of death.

Four per cent of the death certificates coded as asthma were considered to be false positive. This corresponds with previous descriptions in comparable studies $[5,8,9,17]$. Unexpectedly, the authors identified 3\% false negative asthma deaths wrongly coded to another cause, and $9 \%$ false negative asthma deaths coded under adjacent respiratory codes.

The authors were surprised when the current data were compared with other published investigations into the same age group [5,9]. There was no indication of an overestimation of asthma death in the present study. In the majority of patients, the asthma disease was well known and death occurred as a result of well described deterioration in symptoms or an acute asthma attack. Where there was any doubt about the asthma diagnosis as the cause of death, the case was excluded.

From an epidemiological point of view, no false negative certification can be an indication of either overregistration of asthma deaths in the national mortality databases, or collection of a sample of certificates of death due to other respiratory causes that is too small to assess rare causes of death [13].

The authors found that the number of false negative certifications varied over time and increased with increasing autopsy frequency. In the case of an autopsy, which in Denmark consists of both a gross inspection of all organs and a histological examination of relevant organs, the pathologists describe what they find. In patients who died as a result of asthma, the pathologist often finds: 1) mucus plugs in the small airways; 2) emphysema; 3) bronchitis (inflammation, oedema, eosinophils); and 4) bronchial muscular hypertrophy, glandular hyperplasia, and basement membrane thickening. In the current study, the performance of an autopsy did not always result in correct statistical coding. The pathologist had correctly certified that the autopsy was consistent with asthma, and had stated the result of the histological examination, i.e., emphysema and/or bronchitis. Many of these asthma deaths were coded as due to these diagnoses.

The autopsy frequency in the study period decreased due to changes in legislation (1987) and in attitude. An exception was the last 2 yrs of the study period, where only three asthma deaths were identified. However, there was no change in the way autopsies were performed or reported during the study period. The number of false negative coded asthma deaths has decreased since 1988.

The authors have not been able to identify any published studies where the influence of a variation in autopsy frequency is discussed.

An advantage of this study is that the authors had the possibility of examining trends within a given ICD period and later including the latest available year from which data could be collected. Many countries introduced ICD-9 in 1979 [1, 10]. This study has again highlighted the difficulties in ensuring accurate certification of the cause of death, even in children and young adults [11]. The potential for inaccuracies in national mortality statistics has to be underlined.

Further clarification of trends in asthma mortality and circumstances leading to death as a result of asthma may be elucidated by establishment of national medical audit groups and by international mortality comparisons of well described asthma deaths and suspected asthma deaths.

There are still considerable differences in the reported mortality rates in different countries, even among children and young adults [2]. Changes in classification of deaths from asthma in revisions of ICD, inaccuracies in certification and statistical coding of death certificates, and changes in diagnostic criteria do not appear to be sufficient explanation for these differences [2].

Most countries have failed to decrease asthma mortality. In many countries, it has been observed that there has been an increase in the prevalence of asthma in children and young adults, which may partly explain the unchanged pattern of asthma mortality observed in some countries [2, $10]$.

Since 1985, the recommended approach in the management of asthma in children and young adults in Denmark has been to use inhaled corticosteroids as the primary therapy and to use $\beta_{2}$-agonist bronchodilators as rescue medications on an as-needed basis. Many of the paediatric departments introduced inhaled corticosteroids to paediatric patients with severe symptoms of asthma in the beginning of the 1980s. The mortality rate has decreased since 1988 , but factors such as the aetiology of asthma, compliance and education must also have contributed to the change in the death rate.

In conclusion, asthma mortality in Denmark increased in adolescents during 1973-1987 and decreased from 1988 to 1994. The trend analysis must be considered to be highly representative of the true rate of death from asthma in Denmark. Even in children and young adults under the age of $20 \mathrm{yrs}$, validity problems still make comparison between countries difficult; even interpretation of national trends requires caution.

\section{References}

1. Jackson R, Sears MR, Beaglehole R, Rea HH. International trends in asthma mortality: 1970 to 1985 . Chest 1988; 94: 914-918.

2. National Heart, Lung and Blood Institute, National Institutes for Health. Global Strategy for Asthma Management and Prevention: NHLBI/WHO Workshop Report. Bethesda, Maryland, USA: National Institutes of Health. 1995.

3. Evans R, Mullaly DI, Wilson RW, et al. National trends in the morbidity and mortality of asthma in the US. Chest 1987; $91: 65 \mathrm{~s}-74 \mathrm{~s}$. 
4. Bosquet J, Hatton F, Godard P, Michel FB. Asthma mortality in France. J Allergy Clin Immunol 1987; 80: 389-394.

5. Fouchard T, Graff-Lonnevig V. Asthma mortality in Swedish children and young adults 1973-88. Allergy 1994; 49: 616-619.

6. Fletcher HJ, Ibrahim SA, Speight N. Survey of asthma death in the northern region, 1970-85. Arch Dis Childhood 1990; 65: 163-167.

7. Sly RM. Increases in deaths from asthma. Ann Allergy 1984; 53: 20-25.

8. Stewart CJ, Nunn AJ. Are asthma mortality rates changing? Br J Dis Chest 1985; 79: 229-234.

9. Sears MR, Rea HH, De Boer G, et al. Accuracy of certification of deaths due to asthma: a national study. Am J Epidemiol 1986; 124: 1004-1011.

10. Sears MR. Increasing asthma mortality. Fact or artifact? Editorials. J Allergy Clin Immunol 1988; 82: 957960.

11. Vollmer WM, Osborne ML, Buist AS. Uses and limitations of mortality and health care utilization statistics in asthma research. Am J Respir Crit Care Med 1994; 149: S79-S87.

12. Fowles J. Discussant section. Am J Respir Crit Care Med 1994; 149: S88-S90.

13. Kleinbaum DG, Kupper LL, Morgenstern H. Measures of Association. In: Kleinbaum DG, Kupper LL, Morgenstern H, eds. Epidemiologic Research. Principles and Quantitative Methods: New York, USA, Van Nostrand Reinhold Company, 1982; pp. 140-158.

14. Breslow NE, Day NE. Statistical Methods in Cancer Research. Volume II - The Design and Analysis of Cohort Studies. IARC Scientific Publications No. 82. Oxford, UK, Oxford University Press, 1989.

15. Anderson HR, Strachan DP. Asthma mortality in England and Wales, 1979-1989. Lancet 1991; 337: 1357.

16. Campbell MJ, Cogman GR, Holgate ST, Johnston SL. Age specific trends in asthma mortality in England and Wales, 1983-95 results of an observational study. BMJ 1997; 314: 1439-1441.

17. BTA Research Committee. Accuracy of death certificates in bronchial asthma. Thorax 1984; 39: 505-509. 